\title{
Egg rejection and egg recognition mechanisms in Oriental Reed Warblers
}

\author{
Laikun Ma $\mathrm{Ma}^{1,2,3}$ and Wei Liang ${ }^{2^{*}}$ (1)
}

\begin{abstract}
Background: Nest parasitism by cuckoos (Cuculus spp.) results in enormous reproductive failure and forces hosts to evolve antiparasitic strategies, i.e., recognition of own eggs and rejection of cuckoo eggs. There are often sexual conflicts between male and female individuals in the expression of antiparasitic behavior due to the differences in reproductive inputs and division of labor.
\end{abstract}

Methods: By adding a foreign egg made of blue soft clay to the host nest during early incubation period in the field, and by removing several host eggs and adding experimental eggs to control the proportion of two egg types in the nest, we examined egg rejection ability, egg recognition mechanism and sexual difference in egg rejection of the Oriental Reed Warbler (Acrocephalus orientalis), one of the major hosts of Common Cuckoos (Cuculus canorus).

Results: Our results indicated that Oriental Reed Warblers can recognize and reject nearly 100\% (73/75) of the nonmimetic eggs made of blue soft clay, and they could reject foreign eggs with 100\% accuracy, regardless of the ratio of experimental eggs and its own eggs in the nest. Furthermore, all cases of egg rejections recorded by videos were only carried out by females.

Conclusions: Oriental Reed Warblers have a high egg recognition ability and show a true recognition mechanism. Only female warblers perform egg rejection, suggesting that the sex for host egg incubation seems to play an important role in the evolution of egg recognition mechanisms.

Keywords: Cuckoo parasitism, Discordancy, Egg recognition mechanism, Egg rejection, True recognition

\section{Background}

Avian brood parasitism is a specific reproductive behavior in which parasitic birds lay eggs in the nests of other birds (hosts), transferring the costs of incubation and raising offspring to the host (Payne 1977). Successful nest parasitism results in significant reproductive losses to the host, prompting the host to evolve a range of antiparasitic strategies (Davies 2000; Soler 2014). Egg recognition and egg rejection are among the most common antiparasitic strategies and are important indicators of host adaptation to nest parasitism (Davies and

*Correspondence: liangwei@hainnu.edu.cn

${ }^{2}$ Ministry of Education Key Laboratory for Ecology of Tropical Islands, College of Life Sciences, Hainan Normal University, Haikou 571158, China

Full list of author information is available at the end of the article
Brooke 1989a; Moksnes et al. 1991b; Soler et al. 2017; Yang et al. 2020, 2021). For example, in cuckoo parasitic systems, bird species lacking a history of cuckoo parasitism has low or no egg recognition ability, whereas cuckoo hosts often have varied egg recognition abilities (Davies and Brooke 1989a, b; Moksnes et al. 1991a, b). Egg recognition ability acquired by hosts can be maintained for a considerable period of time (Peer et al. 2007, 2011; Yang et al. 2014b; Yi et al. 2020), even in the absence of cuckoo brood parasitism (Honza et al. 2004; Lahti 2005, 2006). However, the level of host egg recognition ability is subject to variation under different parasitic pressure and coevolutionary time, with inter- and intra-specific variations in recognition and rejection of foreign eggs among hosts in the same area or among geographic populations of the same host (Brooke et al. original author(s) and the source, provide a link to the Creative Commons licence, and indicate if changes were made. The images or other third party material in this article are included in the article's Creative Commons licence, unless indicated otherwise in a credit line to the material. If material is not included in the article's Creative Commons licence and your intended use is not permitted by statutory regulation or exceeds the permitted use, you will need to obtain permission directly from the copyright holder. To view a copy of this licence, visit http://creativecommons.org/licenses/by/4.0/. The Creative Commons Public Domain Dedication waiver (http://creativeco mmons.org/publicdomain/zero/1.0/) applies to the data made available in this article, unless otherwise stated in a credit line to the data. 
1998; Lindholm and Thomas 2000; Moskát et al. 2002, 2012; Li et al. 2016; Liang et al. 2016).

In general, the process of egg rejection by host consists of at least three steps: first, the recognition of foreign eggs; second, the decision to discard eggs or not; and last, the rejection of foreign eggs (Soler et al. 2012, 2017). There are two plausible views on the mechanism of host recognition of foreign eggs; one is templatebased recognition, also known as true recognition (Rothstein 1974, 1975; Hauber and Sherman 2001), in which the host knows the characteristics of its own eggs through inheritance or learning and recognizes foreign eggs, independent of whether its own eggs are present or predominant in the nest (i.e., Moskát and Hauber 2007; Moskát et al. 2010; Yi et al. 2020). The second is recognition by discordancy, the simplest form of egg recognition, which determines the least similar eggs as foreign based on the discordancy of egg types within the nest (Rensch 1925; Rothstein 1974, 1975; Davies and Brooke 1989a; Yang et al. 2014c).

Additionally, sex conflict can occur in antiparasitic behavior due to differences in the role division between males and females during breeding (Požgayová et al. 2009; Trnka and Prokop 2010; Trnka et al. 2013), with males showing more aggressive behavior towards nest invaders (Montgomerie and Weatherhead 1988). A study by Li et al. (2015) on Oriental Reed Warblers (Acrocephalus orientalis) found that males were more aggressive in nest defense, while females were more likely to detect intruders. Studies of Great Reed Warblers (Acrocephalus arundinaceus) found that males were primarily responsible for territory defense, while females were responsible for incubation and egg recognition (Požgayová et al. 2009; Trnka and Prokop 2010). Another study of Ashy-throated Parrotbills (Paradoxornis alphonsianus) with egg color polymorphism found that both males and females have egg recognition ability, but they may use different recognition mechanisms (Liang et al. 2012).

The Oriental Reed Warbler, a favorite host of Common Cuckoos (Cuculus canorus) (hereafter cuckoos) in China, is at a high stage of co-evolution with cuckoos (Yang et al. 2014a; Li et al. 2016; Ma et al. 2018a). Egg recognition ability of Oriental Reed Warblers is known to be high, and the Common Cuckoo has formed a highly mimetic reed warbler clade (gens) in the local population (Li et al. 2016; Yang et al. 2016, 2017). Previous work showed that only females incubate eggs, while males are responsible for guarding nearby during the incubation period (Lotem et al. 1992). In this study, we tested egg rejection and egg recognition mechanism of Oriental Reed Warblers by controlling the ratio of experimental eggs and its own eggs in its nests. In addition, we explored whether there were sexual differences in egg recognition by Oriental Reed Warblers.

\section{Methods \\ Study area}

The study area is located in the Yongnian Depressional Wetland $\left(36^{\circ} 40^{\prime}-36^{\circ} 41^{\prime} \mathrm{N}, 114^{\circ} 41^{\prime}-114^{\circ} 45^{\prime} \mathrm{E}\right)$ in Yongnian County, Hebei Province, China, which is a natural depression in the alluvial plain of the Fuyang River. The Yongnian Depressional Wetland has a welldeveloped water system, numerous tributaries, and yearround waterlogging, at an altitude of only $40.3 \mathrm{~m}$. The average annual rainfall is $527.8 \mathrm{~mm}$, mostly in summer, and the average annual temperature is $12.9^{\circ} \mathrm{C}$. The main vegetation type of the wetland is reeds (Phragmites australis), which are interspersed mainly with cattails (Typha latifolia) (Ma et al. 2018a, b).

\section{Sexing of Oriental Reed Warblers in the field}

Both sexes of Oriental Reed Warblers are similar. Our observations show that males have towering head feathers that form a small crest, whereas the females have gentle head feathers and no crest under normal status. This was confirmed with video monitoring showing that incubating parents had the plumage characteristics of females, and it is known that only females incubate eggs (Lotem et al. 1992). In addition, most cases of egg rejection were carried out obviously by incubating individuals without crest. Therefore, the sex of Oriental Reed Warblers can be determined by the pattern of head feathers from videos (Fig. 1).

\section{Egg recognition experiments}

Fieldwork was conducted during the 2016 and 2017 breeding seasons. Because parasitism by common cuckoo usually occurs in the laying or early incubation cycle of host nests for advantage of prior hatching (Geltsch et al. 2016; Wang et al. 2020), egg recognition experiments (one model egg or one real foreign egg was added to an experimental nest) were carried out during the early incubation period, along with video recording using a minicamera. Blue and white model eggs were produced based on Li et al. (2016) using a highly plastic synthetic soft clay to the size of local cuckoo eggs (Table 1). The real eggs used were white unfertilized Budgerigar (Melopsittacus undulatus) eggs coming from artificially bred parrots.

Previous studies have shown that replacement with or direct addition of one experimental egg does not affect the egg rejection of the host (Davies and Brooke 1988), so the egg recognition ability of Oriental Reed Warblers was tested by direct addition of a blue model egg (highly nonmimetic) to the host nest (Fig. 2a). The host's response to 

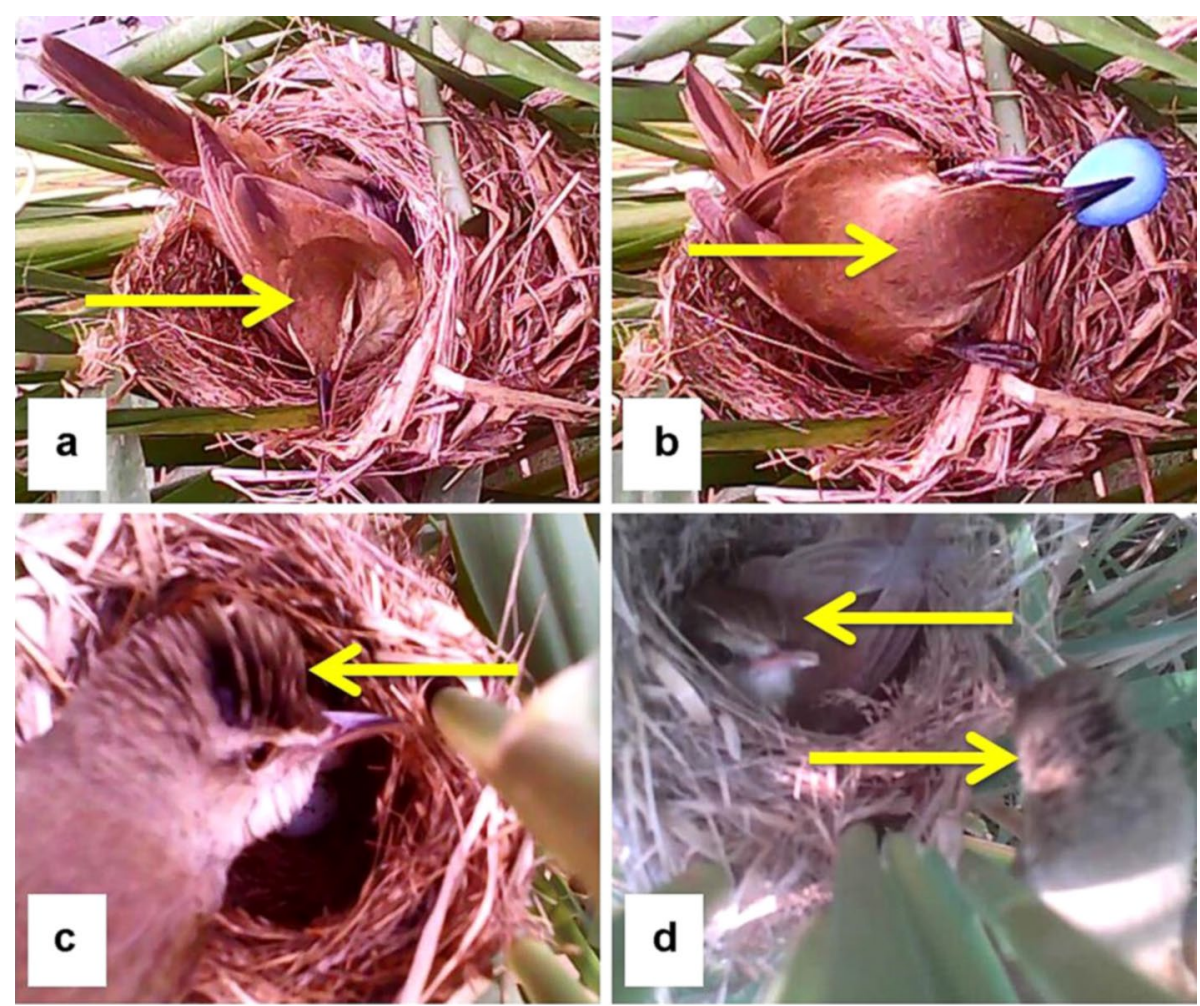

Fig. 1 Comparison of male and female individuals of Oriental Reed Warblers. a refers to a female incubating in the nest; $\mathbf{b}$ refers to a female rejecting a blue model egg; $\mathbf{c}$ refers to a male checking the nest and $\mathbf{d}$ refers to a female incubating and a male checking the nest. Yellow arrows show the top of the head of both sexes, with the female being flat and the male having a crest

Table 1 Parameters of cuckoo and experimental eggs used in this study

\begin{tabular}{lllll}
\hline Egg type & Egg mass $(\mathbf{g})$ & Egg length $(\mathbf{m m})$ & Egg width $(\mathbf{m m})$ & $\begin{array}{l}\text { Sample } \\
\text { size }(\boldsymbol{N})\end{array}$ \\
\hline Common Cuckoo egg & $3.05 \pm 0.09$ & $21.80 \pm 0.55$ & $16.22 \pm 0.40$ & 27 \\
White model egg & $4.27 \pm 0.02$ & $21.84 \pm 0.26$ & $15.88 \pm 0.27$ & 15 \\
Blue model egg & $4.29 \pm 0.02$ & $21.92 \pm 0.30$ & $15.77 \pm 0.25$ & 15 \\
\hline
\end{tabular}

experimental eggs within six days was observed (cf. Moksnes et al. 1991b): if the host did not reject the experimental egg within six days, it was recorded as accepted; if the egg was pecked, disappeared or deserted within six days, it was considered rejected. Experimental nests that were predated or destroyed because of bad weather within six days were not counted in the results of the experiment (see also Yang et al. 2019).

\section{Egg recognition mechanism experiments}

In 2017, two sets of experiments were conducted. In the first experiment, the number of Oriental Reed Warbler eggs and experimental eggs were controlled to both be two to maintain the same proportion. Host eggs were removed provisionally so that there were only two, and two experimental eggs were added to the nest. Experimental eggs used either two white model eggs (Fig. 2b) or two white budgerigar eggs (Fig. 2c). This experiment is thus referred to as the $2+2$ experiment. Eggs were observed for 6 days, and the results were recorded. In the second experiment, the number of Oriental Reed Warbler eggs and experimental eggs was controlled to be one and three, respectively. That is, the number of host eggs was controlled to be lower than that of experimental eggs, because Oriental Reed Warblers are likely to abandon the nest when only one egg is left in the nest. This experiment is thus referred to as the $1+3$ experiment. In a pre-experiment, we found that Oriental Reed 

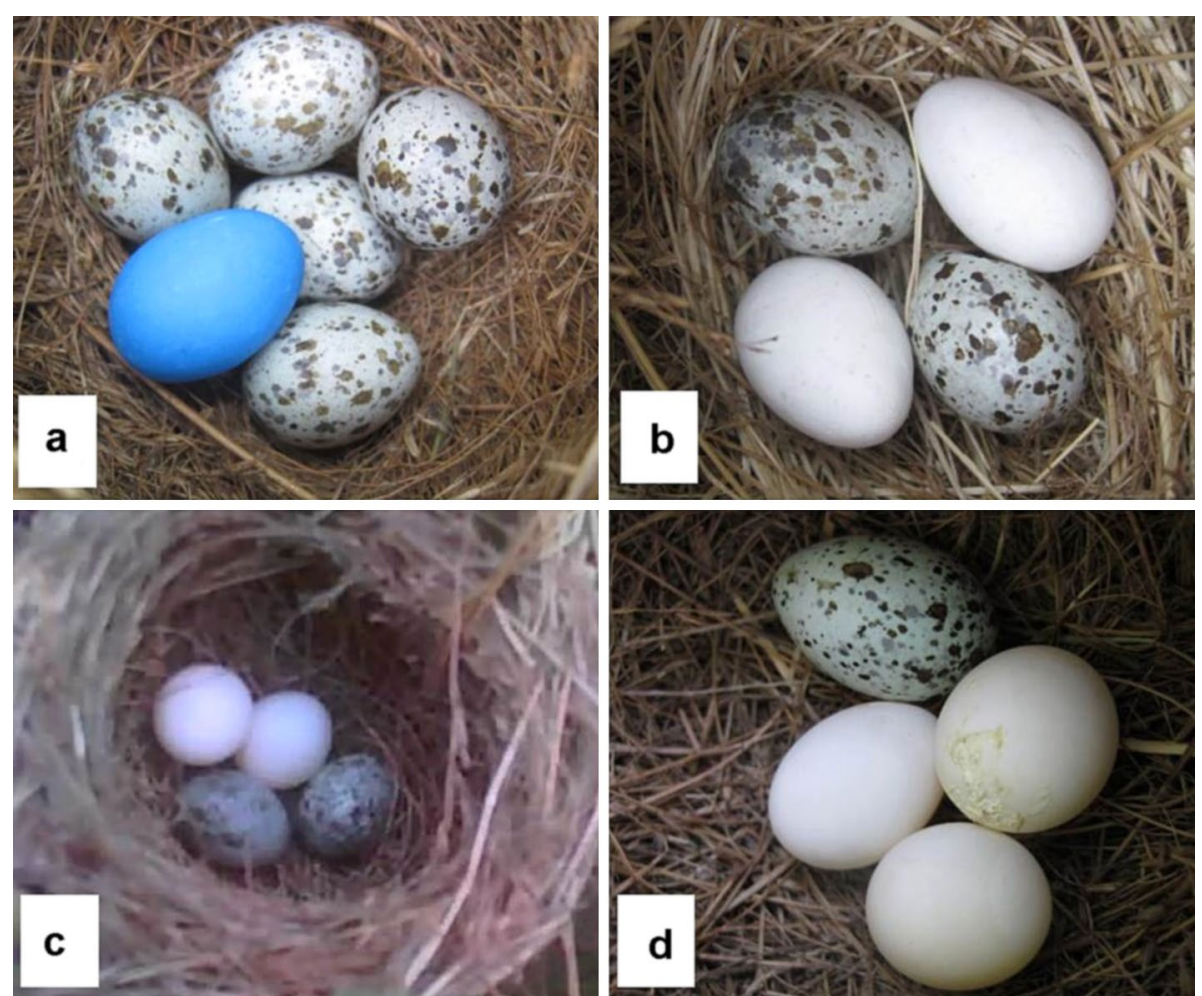

Fig. 2 Egg recognition experiments in nests of the Oriental Reed Warbler. a refers to experimental nest with adding one blue model egg; $\mathbf{b}$ and $\mathbf{c}$ refer to experimental nests with two experimental eggs and two host eggs (i.e., $2+2$ ), while experimental eggs in $\mathbf{b}$ are white model eggs and $\mathbf{c}$ are white budgerigar eggs; $\mathbf{d}$ refers to experimental nests with one host egg and three budgerigar eggs (i.e., $1+3$ )

Warblers were able to quickly recognize and reject experimental eggs in the $1+3$ experiment, and that the timely return of Oriental Reed Warbler eggs to the nest after the experiment could avoid nest desertion, so we checked the experimental results within half an hour after starting the experiment. The experimental eggs used in the $1+3$ experiment were white budgerigar eggs (Fig. 2d). The recognition mechanism experiment was performed only once for each nest.

\section{Data analyses}

Statistical analyses were performed using IBM SPSS 20.0 for Windows. Fisher's exact test was used for comparison between different probabilities and Wilcoxon rank sum test was used to compare the number of nest-returns and egg checks between males and females before egg rejection. All tests were two-tailed, with a significance level of $P<0.05$, and data are presented in the form of mean \pm standard deviation (mean $\pm \mathrm{SD}$ ).

\section{Results}

\section{Egg rejection}

Egg recognition experiments with non-mimetic blue model eggs were performed in 75 nests of Oriental Reed
Warblers. Blue mode eggs were rejected 73 times and accepted two times, at a rejection rate of $97.3 \%$ (Fig. 3; Additional file 1: Video S1). Furthermore, Oriental Reed

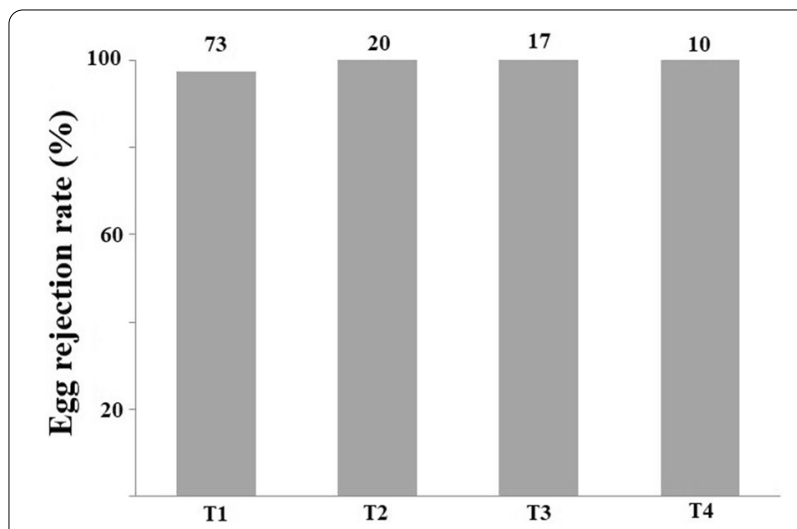

Fig. 3 Egg rejection rates by Oriental reed warblers in egg recognition mechanism experiments. $T 1$ refers to experimental group with one blue model egg added to the nest; $T 2$ refers to experimental group with two host eggs and two white model eggs; $T 3$ refers to experimental group with two host eggs and two white budgerigar eggs; T4 refers to experimental group with one host egg and three white budgerigar eggs. Numbers above bars indicate the sample size 
Warblers rejected all foreign eggs with a rejection rate of $100 \%$ in 11 nests which one foreign egg was added (4 nests with a budgerigar egg, 6 nests with a black-painted Budgerigar egg, and one nest with a Reed Parrotbill (Paradoxornis heudei) egg, Additional file 2: Video S2).

\section{Egg recognition mechanisms}

The $2+2$ experiment was performed in a total of 20 nests with white model eggs and 17 nests with white Budgerigar eggs. The $1+3$ experiment was conducted in 10 nests with white budgerigar eggs. Across all experimental nests, Oriental Reed Warblers were able to accurately recognize its own eggs while rejecting the foreign eggs, with a recognition rate of $100 \%$ and no recognition errors were found, showing the true recognition mechanism (Fig. 3).

\section{Sex roles in egg recognition}

Egg rejections were recorded on videos in 30 nests lasting about two hours until the power run out, including 19 nests for recognition mechanism experiments, and 11 nests in which one foreign egg was added for egg recognition experiments. All eggs were ejected and completed by female Oriental Reed Warblers, 16 of which had males returning and checking eggs prior to egg rejection. Females had significantly more numbers of returning the nest (Female: $8.10 \pm 8.38$, Male: $0.87 \pm 1.33, N=30$ ) and checking eggs (Female: $56.33 \pm 67.01$, Male: $1.70 \pm 3.23$, $N=30$ ) than males for all nests before egg rejection $\left(Z_{1}=-4.643, P_{1}<0.01 ; Z_{2}=-4.585, P_{2}<0.01\right)$.

\section{Discussion}

Our study found that Oriental Reed Warblers rejected non-mimetic experimental eggs with $100 \%$ accuracy, regardless of the ratio of experimental eggs and its own eggs in the nest, presenting a true recognition mechanism. Additionally, all eggs were rejected with ejection without observed recognition errors and only females of Oriental Reed Warblers rejected experimental eggs.

Egg recognition is one of the most effective means for hosts to combat parasitism, but even different geographic populations of the same host can exhibit significant geographic variation in the recognition and rejection of foreign eggs due to differences in the history of co-evolution and reciprocal pressure (Brooke et al. 1998; Lindholm and Thomas 2000; Moskát et al. 2002, 2012; Li et al. 2016; Liang et al. 2016). In this study, Oriental Reed Warblers, as one of the most common hosts of cuckoos, have a nearly $100 \%$ rejection rate for non-mimetic eggs, similar to the population in northeastern China, which rejected both blue $(n=15)$ and white $(n=24)$ model eggs at a rate of $100 \%$ (Wang et al. 2021) and the Japanese population (94\%, $n=33$; Lotem et al. 1995), suggesting that the Oriental Reed Warbler possesses an extremely strong egg recognition ability. However, egg rejection of another population of Oriental Reed Warblers (Li et al. 2016, 2020) was slightly lower than that of this population, possibly because the material and size of experimental eggs used may influence on the host's completion of egg rejection (Roncalli et al. 2017; Li et al. 2020).

In examination of recognition mechanisms of the Oriental Reed Warbler, we found that regardless of the ratio of warbler eggs and experimental eggs in the nest, the Oriental Reed Warbler was able to recognize $100 \%$ of experimental eggs and reject them quickly, suggesting that the Oriental Reed Warbler uses template memory to recognize foreign eggs and shows a true recognition mechanism. Template recognition is a ubiquitous recognition mechanism in hosts, and discordancy recognition has not been found in isolated cases to date, but often coexists with template recognition (Lyon 2007; Moskát et al. 2014; Yang et al. 2014c; Wang et al. 2015). Moskát et al. (2010) found that the European Great Reed Warbler uses both template and discordancy recognition, which was unlike the present results. This may be due to differences in geographic factors that cause the two hosts to be at different stages of coevolution with cuckoos, as was found in a study of European Great Reed Warblers and the Japanese Oriental Reed Warblers, where the two were clearly at different stages of coevolution (Moskát et al. 2012). Another possible reason is that the two studies used different experimental eggs, with the degree of egg mimicry being an important factor in host recognition and rejection (Davies and Brooke 1988; Stokke et al. 2004; Antonov et al. 2006). Moskát et al's (2010) experiments used the host's own real egg painted with spots as experimental eggs, and the similarity between the two egg types may have led to difficulties in host recognition, e.g., the rejection rate of a single experimental egg by the host in the experiment was only $32 \%(6 / 19)$, which was much lower than the previously reported rejection rate of non-mimetic eggs by the same host species (Moskát et al. 2008). Clearly, these need to be confirmed further in the future to use similar and mimetic experimental eggs to examine the recognition mechanism in warbler hosts.

The egg rejection behaviors recorded on videos in this study all occurred in females, which may be due to the differential division of roles between male and female individuals during reproduction (Požgayová et al. 2009; Trnka and Prokop 2010). Oriental Reed Warbler males are not involved in egg incubation, but in territory defense and nest defense (Li et al. 2015); whereas females are responsible for egg incubation. By contrast, in ashy-throated parrotbills, males and females take turns incubating eggs, and both males and females have egg recognition ability but use different recognition 
mechanisms to reject eggs to ensure maximum benefit (Liang et al. 2012; Yang et al. 2014c). Therefore, the sex for incubation seems to play an important role in the evolution of host egg recognition. Such sexual difference is also present in the anti-parasitic behavior of the host's nest defense (Požgayová et al. 2009; Trnka and Prokop 2010; Li et al. 2015).

\section{Conclusions}

In summary, the present study showed that Oriental Reed Warblers possess a high degree of egg recognition ability and employs a true recognition mechanism. In addition, all egg rejection is conducted by female individuals with ejection. The sexual difference for host nest defense and egg incubation seems to play an important role in the evolution of egg recognition mechanisms, which needs more investigations in future work.

\section{Supplementary Information}

The online version contains supplementary material available at https://doi. org/10.1186/s40657-021-00283-4.

Additional file 1: Video S1. A blue model egg rejected by the Oriental Reed Warbler host in the egg recognition experiment.

Additional file 2: Video S2. A Reed Parrotbill egg rejected by the Oriental Reed Warbler host in the egg recognition experiment.

\section{Acknowledgements}

We thank the Forestry Bureau of Yongnian County, Hebei Province, China, for permission to undertake this study. We are grateful to Canchao Yang and Jianping Liu for help with statistical analyses, Xiaodong Rao and Jianwei Zhang for assistance with fieldwork.

\section{Authors' contributions}

WL designed the study; LM carried out field experiments, performed statistical analyses and wrote the draft manuscript. WL revised and improved the manuscript. Both authors read and approved the final manuscript.

\section{Funding}

This work was supported by the National Natural Science Foundation of China (No. 31970427 to WL and 32101242 to LM) and by the Open Foundation of Hebei Key Laboratory of Wetland Ecology and Conservation (hklk201903 to $L M)$ and the Natural Science Foundation of Hebei Province of China (C2020101002 to LM).

\section{Data availability statement}

The data that supports the findings of this study are available in the supplementary material of this article.

\section{Declarations}

\section{Ethics approval and consent to participate}

The experiments comply with the current laws of China. Experimental procedures were in agreement with the Animal Research Ethics Committee of Hainan Provincial Education Centre for Ecology and Environment, Hainan Normal University (permit no. NECEE-2012-003).

\section{Competing interests}

The authors declare that they have no competing interests.

\section{Author details}

${ }^{1}$ Department of Biology and Food Science, Hebei Normal University for Nationalities, Chengde 067000, China. ${ }^{2}$ Ministry of Education Key Laboratory for Ecology of Tropical Islands, College of Life Sciences, Hainan Normal University, Haikou 571158, China. ${ }^{3}$ Hebei Key Laboratory of Wetland Ecology and Conservation, Hengshui University, Hengshui 053000, China.

Received: 19 June 2021 Accepted: 7 September 2021

Published online: 21 September 2021

\section{References}

Antonov A, Stokke BG, Moksnes A, Røskaft E. Egg rejection in marsh warblers (Acrocephalus palustris) heavily parasitized by common cuckoos (Cuculus canorus). Auk. 2006;123:419-30.

Davies NB. Cuckoos, cowbirds and other cheats. London:T \& AD Poyser; 2000.

Davies NB, deL Brooke M. Cuckoos versus reed warblers: adaptations and counteradaptations. Anim Behav. 1988;36:262-84.

Davies NB, deL Brooke M. An experimental study of co-evolution between the cuckoo, Cuculus canorus, and its hosts. I. Host egg discrimination. J Anim Ecol. 1989a;58:207-24.

Davies NB, deL Brooke M. An experimental study of co-evolution between the cuckoo, Cuculus canorus, and its hosts. II. Host egg markings, chick discrimination and general discussion. J Anim Ecol. 1989b;58:225-36.

deL Brooke M, Davies NB, Noble DG. Rapid decline of host defences in response to reduced cuckoo parasitism: behavioural flexibility of reed warblers in a changing world. Proc R Soc Lond B-Biol Sci. 1998;265:1277-82.

Geltsch N, Bán M, Hauber ME, Moskát C. When should common cuckoos Cuculus canorus lay their eggs in host nests? Bird Study. 2016;63:1-6.

Hauber ME, Sherman PW. Self-referent phenotype matching: theoretical considerations and empirical evidence. Trends Neurosci. 2001;24:609-16.

Honza M, Procházka P, Stokke BG, Moksnes A, Røskaft E, Čapek M, et al. Are blackcaps current winners in the evolutionary struggle against the common cuckoo? J Ethol. 2004;22:175-80.

Lahti DC. Evolution of bird eggs in the absence of cuckoo parasitism. Proc Natl Acad Sci USA. 2005;102:18057-62.

Lahti DC. Persistence of egg recognition in the absence of cuckoo brood parasitism: pattern and mechanism. Evolution. 2006;60:157-68.

Li D, Wei H, Zhang Z, Liang W, Stokke BG. Oriental reed warbler (Acrocephalus orientalis) nest defence behaviour towards brood parasites and nest predators. Behaviour. 2015;152:1601-21.

Li D, Zhang Z, Grim T, Liang W, Stokke BG. Explaining variation in brood parasitism rates between potential host species with similar habitat requirements. Evol Ecol. 2016;30:905-23.

Li D, Li X, Zhang Y, Guan S, Ruan Y. Contrasting effects of egg size and appearance on egg recognition and rejection response by Oriental reed warblers. Ecol Evol. 2020;10:11523-34.

Liang W, Yang C, Antonov A, Fossøy F, Stokke BG, Moksnes A. Sex roles in egg recognition and egg polymorphism in avian brood parasitism. Behav Ecol. 2012;23:397-402.

Liang W, Møller AP, Stokke BG, Yang C, Kovařík P, Wang H, et al. Geographic variation in egg ejection rate by great tits across 2 continents. Behav Ecol. 2016;27:1405-12.

Lindholm AK, Thomas RJ. Differences between populations of reed warblers in defences against brood parasitism. Behaviour. 2000;137:25-42.

Lotem A, Nakamura H, Zahavi A. Rejection of cuckoo eggs in relation to host age: a possible evolutionary equilibrium. Behav Ecol. 1992;3:128-32.

Lotem A, Nakamura H, Zahavi A. Constraints on egg discrimination and cuckoo-host co-evolution. Anim Behav. 1995;49:1185-209.

Lyon B. Mechanism of egg recognition in defenses against conspecific brood parasitism: American coots (Fulica americana) know their own eggs. Behav Ecol Sociobiol. 2007;61:455-63.

Ma L, Yang C, Liang W. Hawk mimicry does not reduce attacks of cuckoos by highly aggressive hosts. Avian Res. 2018a;9:35.

Ma L, Yang C, Liu J, Zhang J, Liang W, Møller AP. Costs of breeding far away from neighbors: Isolated host nests are more vulnerable to cuckoo parasitism. Behav Proc. 2018b;157:327-32.

Moksnes A, Røskaft E, Braa AT. Rejection behavior by common cuckoo hosts towards artificial brood parasite eggs. Auk. 1991a;108:348-54. 
Moksnes A, Røskaft E, Lampe HM. Behavioural responses of potential hosts towards artificial cuckoo eggs and dummies. Behaviour. 1991b:116:64-89.

Montgomerie RD, Weatherhead PJ. Risks and rewards of nest defence by parent birds. Q Rev Biol. 1988;63:167-87.

Moskát C, Hauber ME. Conflict between egg recognition and egg rejection decisions in common cuckoo (Cuculus canorus) hosts. Anim Cogn. 2007; 10:377-86.

Moskát C, Szentpéteri J, Barta Z. Adaptations by great reed warblers to brood parasitism: A comparison of populations in sympatry and allopatry with the common cuckoo. Behaviour. 2002;139:1313-29.

Moskát C, Hansson B, Barabás L, Bártol I, Karcza Z. Common cuckoo Cuculus canorus parasitism, antiparasite defence and gene flow in closely located populations of great reed warblers Acrocephalus arundinaceus. J Avian Biol. 2008;39:663-71.

Moskát C, Bán M, Székely T, Komdeur J, Lucassen RW, van Boheemen LA, et al. Discordancy or template-based recognition? Dissecting the cognitive basis of the rejection of foreign eggs in hosts of avian brood parasites. $J$ Exp Biol. 2010;213:1976-83.

Moskát C, Takasu F, Munoz AR, Nakamura H, Bán M, Barta Z. Cuckoo parasitism on two closely-related Acrocephalus warblers in distant areas: A case of parallel coevolution. Chin Birds. 2012;3:320-9.

Moskát C, Zölei A, Bán M, Elek Z, Tong L, Geltsch N, et al. How to spot a stranger's egg? A mimicry-specific discordancy effect in the recognition of parasitic eggs. Ethology. 2014;120:616-26.

Payne RB. The ecology of brood parasitism in birds. Annu Rev Ecol Evol Syst. 1977;8:1-28.

Peer BD, Rothstein SI, Delaney KS, Fleischer RC. Defence behaviour against brood parasitism is deeply rooted in mainland and island scrub-jays. Anim Behav. 2007;73:55-63.

Peer BD, Kuehn MJ, Rothstein SI, Fleischer RC. Persistence of host defence behaviour in the absence of avian brood parasitism. Biol Lett. 2011;7:670-3.

Požgayová M, Procházka P, Honza M. Sex-specific defence behaviour against brood parasitism in a host with female-only incubation. Behav Proc. 2009;81:34-8.

Rensch B. Verhalten von SingvöelnbeiÄderung des Geleges. Ornithol Monatsber. 1925;33:169-73.

Roncalli G, Ibáñez-Álamo D, Soler M. Size and material of model parasitic eggs affect the rejection response of western Bonelli's warbler Phylloscopus bonelli. Ibis. 2017;159:113-23.

Rothstein SI. Mechanisms of avian egg recognition: Possible learned and innate factors. Auk. 1974;91:796-807.

Rothstein SI. Mechanism of egg recognition: Do birds know their own eggs? Anim Behav. 1975;23:268-78.

Soler M. Long-term coevolution between avian brood parasites and their hosts. Biol Rev. 2014:89:688-704.
Soler M, Fernández-Morante J, Espinosa F, Martín-Vivaldi M. Pecking but accepting the parasitic eggs may not reflect ejection failure: the role of motivation. Ethology. 2012;118:662-72.

Soler M, Ruiz-Raya F, Roncalli G, Ibáñez-Álamo JD. Relationships between egg-recognition and egg-ejection in a grasp-ejector species. PLOS ONE. 2017;12:e0166283.

Stokke BG, Rudolfsen G, Moksnes A, Røskaft E. Rejection of conspecific eggs in chaffinches: the effect of age and clutch characteristics. Ethology. 2004:110:459-70.

Trnka A, Prokop P. Does social mating system influence nest defence behaviour in great reed warbler (Acrocephalus arundinaceus) males? Ethology. 2010;116:1075-83.

Trnka A, Požgayová M, Samaš P, Honza M. Repeatability of host female and male aggression towards a brood parasite. Ethology. 2013;119:907-17.

Wang L, Yang C, Møller AP, Liang W, Lu X. Multiple mechanisms of egg recognition in a cuckoo host. Behav Ecol Sociobiol. 2015;69:1761-7.

Wang L, Yang C, He G, Liang W, Møller AP. Cuckoos use host egg number to choose host nests for parasitism. Proc R Soc B. 2020;287:20200343.

Wang L, He G, Zhang Y, Ma J, Liang W. Cryptic eggs are rejected less frequently by a cuckoo host. Anim Cogn. 2021. https://doi.org/10.1007/ s10071-021-01507-2.

Yang C, Li D, Wang L, Liang G, Zhang Z, Liang W. Geographic variation in parasitism rates of two sympatric cuckoo hosts in China. Zool Res. 2014a;35:67-71.

Yang C, Liu Y, Zeng L, Liang W. Egg color variation, but not egg rejection behavior, changes in a cuckoo host breeding in the absence of brood parasitism. Ecol Evol. 2014b;4:2239-46.

Yang C, Møller AP, Røskaft E, Moksnes A, Liang W, Stokkle BG. Reject the odd egg: egg recognition mechanisms in parrotbills. Behav Ecol. 2014C;25:1320-4

Yang C, Wang L, Liang W, Møller AP. Egg recognition as antiparasitism defence in hosts does not select for laying of matching eggs in parasitic cuckoos. Anim Behav. 2016;122:177-81.

Yang C, Wang L, Liang W, Møller AP. How cuckoos find and choose host nests for parasitism. Behav Ecol. 2017;28:859-65.

Yang C, Wang L, Liang W, Møller AP. High egg rejection rate in a Chinese population of grey-backed thrush (Turdus hortulorum). Zool Res. 2019:40:226-30.

Yang C, Wang L, Cheng S-J, Hsu Y-C, Møller AP, Liang W. Model eggs fail to detect egg recognition in host populations after brood parasitism is relaxed. Front Zool. 2020;17:14.

Yang C, Huang J, Liang W, Møller AP. Absence of anti-parasitic defenses in an Asian population of the magpie, a regular host of the great spotted cuckoo in Europe. Curr Zool. 2021;67:345-7.

Yi T, Sun YH, Liang W. Egg rejection and egg recognition mechanism of chestnut thrushes (Turdus rubrocanus). Behav Proc. 2020:178:104158.

Ready to submit your research? Choose BMC and benefit from

- fast, convenient online submission

- thorough peer review by experienced researchers in your field

- rapid publication on acceptance

- support for research data, including large and complex data types

- gold Open Access which fosters wider collaboration and increased citations

- maximum visibility for your research: over $100 \mathrm{M}$ website views per year

At BMC, research is always in progress.

Learn more biomedcentral.com/submissions 